$4^{\text {th }}$ International Conference on Industrial Engineering and Industrial Management

XIV Congreso de Ingeniería de Organización

Donostia - San Sebastián, September $8^{\text {th }}-10^{\text {th }} 2010$

\title{
Estudio empírico de las prácticas de Responsabilidad Social Corporativa en la minería de áridos en Cataluña
}

\section{Empirical study of CSR practices in the aggregate mining in Catalonia}

\author{
Carla Vintró Sánchez y Jordi Fortuny Santos \\ Dpto. de Organización de Empresas. Escuela Politécnica Superior de Ingeniería de Manresa. \\ Universidad Politécnica de Catalunya. Av. Bases de Manresa, 61-73, 08242. Manresa (Barcelona). \\ carla.vintro@upc.edu,Jordi.Fortuny@upc.edu
}

Fecha de recepción: 8-9-2010

Fecha de aceptación: 27-10-2010

Resumen: El objetivo de este trabajo es analizar la situación de la responsabilidad social corporativa (RSC) en la minería de áridos de Cataluña. A partir de la información obtenida mediante cuestionarios se ha analizado la experiencia en sistemas de gestión, la adopción de prácticas de RSC, la aplicación de procedimientos de RSC y la utilización de sistemas de indicadores de la RSC. Los resultados muestran un incipiente interés por la RSC. Destacan (en positivo) el compromiso con el entorno y la preocupación por los stakeholders internos de la empresa. Uno de los aspectos que debe mejorarse es la gestión de las acciones de RSC.

Palabras clave: RSC (responsabilidad social corporativa), ética, sostenibilidad, sistemas de gestión.

Abstract: The aim of this work is to analyze the situation of corporate social responsibility (CSR) in the aggregate mining in Catalonia. From the information obtained with questionnaires we analyzed the experience that companies have on management systems, the adoption of CSR practices, the application of CSR procedures, and the utilization of CSR indicators. The results reveal an incipient interest for CSR. In a prominent position, the compromise with the environment and the concern for internal stakeholders stand out. CSR management should be improved.

Keywords: CSR (corporate social responsibility), ethics, sustainability, management systems.

\section{Introducción}

Durante las últimas décadas se han experimentado profundos cambios en el entorno competitivo a la vez que la conciencia moral de las organizaciones ha ido en aumento. Los aspectos éticos y de sostenibilidad han ganado importancia, y a los objetivos tradicionales de maximizar las ventas (generar valor para el consumidor) y la rentabilidad (generar valor para los accionistas) se han unido los de responsabilidad social.Todo ello ha derivado en la integración de la triple cuenta de resultados: económicos, sociales y medioambientales, que constituye la base de la Responsabilidad Social Corporativa (en adelante RSC).

Este nuevo enfoque empresarial toma especial relevancia en el sector minero, que constituye una de las actividades económicas más antiguas de la humanidad. Su evolución ha estado en gran parte correla- cionada con el progreso de las civilizaciones, y actualmente desarrolla un papel fundamental en la sociedad moderna y en los procesos industriales (GRI, 2005), puesto que aporta materias primas y fuentes de energía. Sin embargo, tradicionalmente esta actividad ha sido percibida como responsable de un elevado impacto en el entorno (Jenkins, 2004) y en la salud de sus trabajadores (Navarro y Dinis, 2003).

Las actuaciones del sector minero en el ámbito de la gestión ética y sostenible están desde hace ya bastantes años en el punto de mira de muchos grupos de interés (Törey, 2004). Inicialmente la contribución con la sociedad, en términos éticos, residía básicamente en acciones filantrópicas asociadas a caridad, pero últimamente se han empezado a incluir temas de responsabilidad social y medioambiental en las agendas de la industria minera (Cowell et al., 1999), y distintas empresas mineras han iniciado proceso de inversión social (Mogrovejo et al., 2007). 
Tanta es la importancia de la RSC en la minería, que distintos organismos han incentivado su aplicación. Un ejemplo de ello es el Consejo Internacional de Minería y Metales (ICMM) que ha promovido el desarrollo sostenible de la minería como fuente de ventaja competitiva, y desde el año 2003, ha fijado 10 principios básicos de buenas prácticas entre los que se incluye la gestión ética, el desarrollo sostenible, y la contribución en el desarrollo social y económico de las comunidades en las que operan las explotaciones (ICMM, 2005).

En definitiva, la aplicación de la RSC en las actividades mineras debe considerarse un medio de conciliación entre los intereses de la industria minera, el gobierno y la sociedad en general (Guerra, 2004), a la par que una inversión social que permita su propio desarrollo y que mejore su reputación (Fombrun y Shanley, 1990).

Dentro del marco teórico comentado, el presente trabajo de investigación trata de mostrar la difusión de la gestión de RSC entre las explotaciones de áridos de Cataluña. El objetivo específico del mismo es determinar las prácticas predominantes, el nivel de formalización en la medida de los objetivos de responsabilidad social y medioambiental, y la aplicación de sistemas de gestión ética y sostenible. Al objeto de comprobar estos aspectos, se ha planteado un estudio empírico centrado en el sector de los áridos de la región de Cataluña en el que se analiza la opinión y prácticas de las empresas en torno a las cuestiones relacionadas.

\section{Revisión de la literatura}

La RSC puede definirse (Erkoreka, 2006) como una forma de gestionar la empresa, integrada en la estrategia de ésta, que compatibiliza la rentabilidad (visión tradicional de negocio), con actuaciones sociales y medioambientales (visión de desarrollo sostenible).

También puede entenderse como una potente herramienta de gestión (Carroll, 1999) o como un modelo integral de gestión empresarial orientado hacia la excelencia a largo plazo (Galán, 2008), que persigue la formalización de una opinión positiva del cliente sobre la corporación, y que actúa en beneficio de los distintos grupos de interés de la empresa mediante el cumplimiento de obligaciones y compromisos legales y éticos.

Las investigaciones existentes en la literatura sobre RSC en la industria minera no son por el momento muy extensas, lo que se explica por la reciente po- pularización de este sistema de gestión. De todas formas, pueden encontrarse diversos estudios que analizan las prácticas adoptadas en distintos países (Deegan y Rankin, 1996; Yakovleva, 2005; Hamann y Kapelus, 2004; Harris, 2007). Distintos autores han estudiado las estrategias de RSC y su relación con las comunidades locales (Kapelus, 2002; Banks, 2006; Jenkins y Yakovleva, 2006) y con los grupos de interés (Hamann, 2004). Otros han analizado en profundidad las razones por las que la RSC es importante para la minería (Walker y Howard, 2002; Jenkins, 2004), y se han concentrado en temas de sostenibilidad (Warhurst, 200 I).

También pueden encontrarse estudios sobre la adherencia de distintas compañías a guías de responsabilidad social como por ejemplo las del GRI (Jenkins y Yakovleva, 2006), además de estudios sobre el grado de publicación de resultados de RSC. Por ejemplo el estudio internacional conducido por KPMG (2008), concluye que el $70 \%$ de una muestra de 54 empresas mineras publica sus resultados de sostenibilidad en memorias específicas o en el informe anual, aunque solamente el $49 \%$ de las empresas españolas que ha definido objetivos sociales y medioambientales utiliza indicadores para la gestión de estos aspectos (Castilla, 2003). En este sentido, Jenkins y Yakovleva (2006) enfatizan que a la hora de adoptar guías de indicadores de RSC debería cuestionarse la extensión de la información de entrada requerida en relación con el tamaño de la empresa.

\section{Metodología}

La información básica de este trabajo procede de un estudio más amplio sobre la aplicación de sistemas de gestión (calidad, medioambiente, seguridad, y responsabilidad social) en la minería de Cataluña.

La investigación se inició con el diseño de un cuestionario, que constituye la base fundamental para la recogida de la información primaria. Se hizo un pretest con una muestra reducida de empresas, para probar la viabilidad del mismo y detectar dificultades en la interpretación de las preguntas.

La versión final del cuestionario incluye 12 preguntas para el apartado concreto de la RSC, que hacen referencia a las prácticas de RSC, a los procedimientos y sistemas de medida de las prácticas de RSC, y a los sistemas de gestión ética y sostenible. Incluye cuestiones abiertas y cuestiones de selección múltiple, y escalas de valoración tipo Likert de cinco puntos.

Para determinar la población objeto de estudio se seleccionó la base de datos del Gremio de Áridos 
de Cataluña (compuesta por 147 empresas), y se llegó a un acuerdo para establecer contacto inicial con las empresas afiliadas. La asociación aglutina un número muy importante de las explotaciones mineras de Cataluña, aproximadamente unas 380 sobre un total de 574 , lo que equivale a más del $60 \%$ de las explotaciones mineras registradas en la comunidad autónoma según datos del "Registro de explotaciones mineras de Cataluña (Directorio del año 2008)" de la Subdirección General de Minas del Gobierno de España. De los datos se deduce que una misma empresa puede operar varias explotaciones. En nuestro trabajo la unidad de análisis es la empresa.

Se escogió el sector de los áridos por ser el más representativo, puesto que se sitúa como primer subsector minero en España (y también en Europa) con un 58,3\% sobre el total de explotaciones. En España, Cataluña es la segunda comunidad con mayor producción, con un 13,3\% de la producción estatal de áridos, según datos publicados en el Informe Anual del Sector de los Áridos para el año 2008.

Todos los cuestionarios se mandaron, junto con una carta de presentación, por correo electrónico y correo postal a la atención del responsable de sistemas de gestión, o en su defecto a la atención del gerente. Previo al envío, se notificó mediante una circular interna y mediante un mail informativo. Después de todo este proceso, se subsanaron las deficiencias detectadas en las respuestas a través de consultas telefónicas y por correo electrónico.

Al terminar el trabajo de campo, el número de cuestionarios válidos recibidos fue de 30, lo que corres- ponde a un 20,4\% de respuesta. En uno de los cuestionarios recibidos no pudieron subsanarse las deficiencias por lo que no se incluyó en la investigación. De las I 6 empresas restantes que desarrollan su actividad en el sector no se logró respuesta. El nivel de respuesta obtenido puede considerarse satisfactorio según las estadísticas disponibles que fijan como normal un porcentaje de entre el 20 y el $40 \%$ para encuestas postales en España (Ortega, 1990), y aconsejan una tasa de respuesta igual o superior al 20\% para unos resultados positivos en las encuestas por correo electrónico (Malhotra y Grover, 1998).

\section{Análisis de resultados}

\section{I. Análisis descriptivo}

En la Tabla I se presenta la ficha técnica del trabajo de campo realizado.

Se ha realizado un análisis descriptivo de las empresas de la muestra en relación a su tamaño (expresado en número de trabajadores y siguiendo el criterio de la Unión Europea en su recomendación 2003/36 I/CE) y experiencia en sistemas de gestión. Para este último aspecto se ha considerado el número de sistemas certificados implantados (calidad, medioambiente, seguridad y/o responsabilidad social corporativa), el año de obtención de la certificación y las intenciones de futuro.

Por su dimensión (Tabla 2), la mayoría de las empresas de la muestra son pymes: un $70 \%$ son pequeñas y un $20 \%$ son medianas.

Tabla 1.

Ficha técnica del trabajo de campo realizado.

\begin{tabular}{|l|l|}
\hline Población: & | 47 empresas con explotaciones de áridos \\
\hline Ámbito geográfico: & Cataluña \\
\hline Unidad muestral: & Empresa \\
\hline Encuestado: & Responsable sistemas de gestión (en su defecto: gerente) \\
\hline Tamaño muestral: & Toda la población \\
\hline Tasa de respuesta: & $20,4 \%$ (30 empresas) \\
\hline Error de muestreo: & $<12 \%$ \\
\hline
\end{tabular}

\begin{tabular}{|l|c|c|}
\hline Detalle: & $\mathbf{N}^{\circ}$ de empresas & \% sobre el total \\
\hline Empresas que constan al registro & 147 & 100 \\
\hline Cuestionarios recibidos durante la prueba piloto & 5 & 3,4 \\
\hline Cuestionarios recibidos después del primer mailing & 15 & 10,2 \\
\hline Cuestionarios recibidos después de un recordatorio & 4 & 2,7 \\
\hline Cuestionarios recibidos después del segundo mailing & 7 & 4,8 \\
\hline Total cuestionarios recibidos & I & 21,1 \\
\hline Cuestionarios no válidos & 30 & 0,7 \\
\hline Total cuestionarios válidos & & 20,4 \\
\hline
\end{tabular}


Tabla 2

\section{Distribución por tamaño y volumen de explotación}

\begin{tabular}{|l|c|}
\hline Distribución por tamaño & Frecuencia \\
\hline $\begin{array}{l}\text { Microempresa }(<10 \text { trabajadores } \\
y<2 \text { millones de } €)\end{array}$ & $21(3 \%)$ \\
\hline $\begin{array}{l}\text { Pequeña empresa }(<50 \text { trabajadores } \\
y<10 \text { millones de } €)\end{array}$ & $6(20 \%)$ \\
\hline $\begin{array}{l}\text { Mediana empresa }(<250 \text { trabajadores } \\
y<43 \text { millones de } €)\end{array}$ & $2(7 \%)$ \\
\hline Grande empresa & $210 \%$ \\
\hline
\end{tabular}

Con respecto a la experiencia en sistemas de gestión, se observa que el 57\% de las empresas tiene implantado un sistema de gestión de la calidad ISO 900I, el 23\% dispone de un sistema de gestión medioambiental ISO I 400 I, y solamente el 3\% ha implantado un sistema de gestión de la seguridad y salud laboral OHSAS I800I.

Únicamente una empresa tiene implantado un sistema de gestión ética y sostenible. Se trata de una empresa de pequeña dimensión que ha implantado la norma UNE 22480 de Gestión Minera Sostenible, y que se sitúa como la primera pedrera sostenible de Cataluña y la tercera española en obtener la certificación otorgada por la Asociación Española de Normalización y Certificación (AENOR).

Un 43\% de las empresas encuestadas dispone de un único sistema de gestión, un 20\% tiene implantados dos sistemas, y ninguna de las empresas de la muestra posee los tres sistemas normalizados considerados. Además, una empresa ha declarado haber implantado un sistema de gestión de la calidad propio y 7 empresas un sistema propio para la gestión de la seguridad y salud laboral. Estos porcentajes se modifican a $27 \%$ empresas con un sistema, $37 \%$ con dos sistemas y $3 \%$ con tres sistemas de gestión implantados.

Los datos obtenidos indican que la dimensión empresarial no es un factor decisivo para la implantación de sistemas de gestión. Este mismo resultado fue alcanzado por Llorens et al. (2002) en un estudio sobre la implantación de elementos relacionados con la dirección, procesos y cultura de pymes y grandes empresas. Los autores no observaron diferencias significativas y concluyeron que el tamaño de la empresa no influye en el proceso de implantación de un sistema de gestión de calidad total (resultado que puede extrapolarse a los sistemas de gestión en general). Aún así, es cierto que existen dificultades in- herentes a la propia dimensión de la empresa, principalmente en el acceso a recursos económicos (Spence, 1999).

En cuanto a la evolución en el número de certificados se observa un crecimiento a lo largo de los años, especialmente en los sistemas de gestión de la calidad y del medioambiente. Probablemente las presiones competitivas por parte de clientes en el primer caso, y el endurecimiento de la legislación medioambiental en el segundo, son dos factores explicativos. La introducción de sistemas de calidad fue la primera en iniciarse y le siguieron los sistemas medioambientales, los de seguridad y salud laboral, y recientemente los de responsabilidad social corporativa. Este orden de aplicación se corresponde con el orden de publicación de los estándares relacionados. En la figura I se muestra el número de certificaciones acumuladas.

Figura I

Evolución en el $n^{\circ}$ de certificaciones

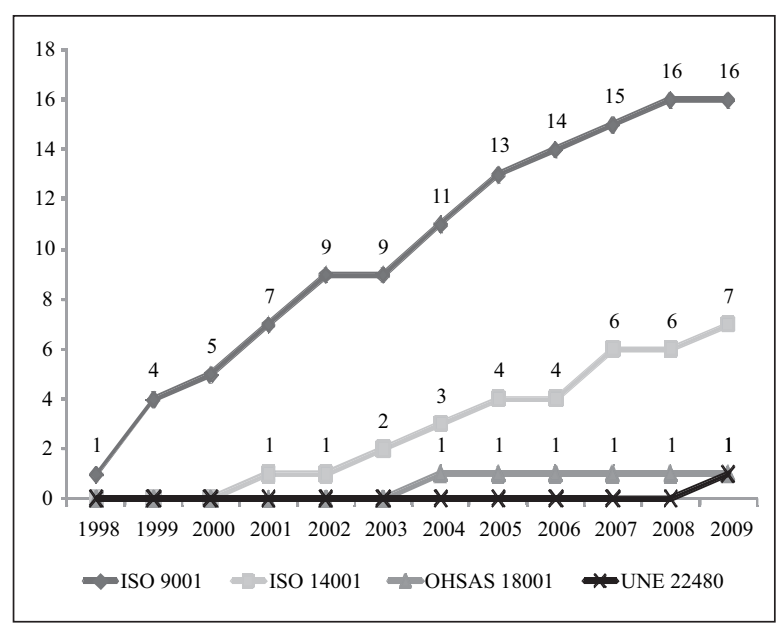

Se aprecia un interés incipiente en seguir trabajando en la implantación y certificación de sistemas de gestión (Figura 2). De las 30 empresas encuestadas, el 83\% tiene intención de implantar un o más sistemas, frente a un 17\% que no plantea estos objetivos en su estrategia para el corto o medio plazo.

La Figura 2 muestra que I I empresas han manifestado su voluntad de implantar un sistema de gestión de RSC en un futuro. Este resultado se alinea con las perspectivas generales de aplicación de sistemas de gestión en el sector, por lo que puede considerarse bastante positivo. Sin embargo, existe la contrapartida (y resultado negativo) que un 52\% de las empresas declara no conocer la norma de gestión ética y sostenible UNE 22480 específica para la minería. 
De cumplirse los objetivos expresados por las empresas, los porcentajes de aplicación de sistemas de gestión ascenderían a un 83\% para los sistemas de calidad ISO 900I, 73\% para los sistemas medioambientales ISO |400I, 50\% para los sistemas de seguridad laboral OHSAS I 800 I y $40 \%$ para la certificación de RSC (porcentajes sobre la muestra considerada).

Figura 2

$\mathrm{N} .^{\circ}$ de empresas interesadas en implantar sistemas de gestión

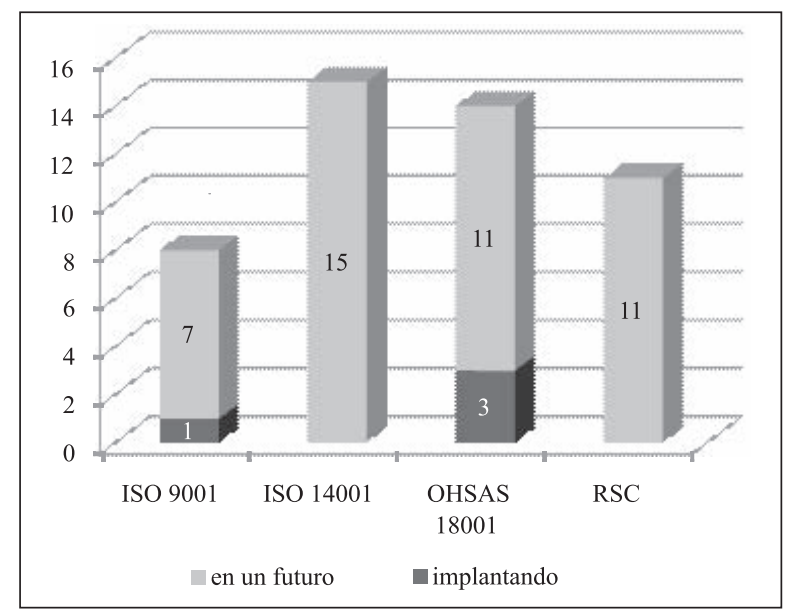

\subsection{Prácticas de RSC}

En una segunda fase se analizan las prácticas de RSC aplicadas por las empresas, el nivel de formalización en los procedimientos de RSC, y el nivel de aplicación de sistemas de medida de los resultados de RSC. La variable utilidad se ha evaluado con una escala de Likert de 5 puntos ( I: muy baja utilidad; 5: utilidad muy alta). Las variables procedimientos y sistemas de medida de los resultados también se han evaluado a través de una escala Likert de 5 puntos ( 1 : no en absoluto; 5: si totalmente). Las prácticas de RSC incluyen 8 categorías que corresponden a las 8 prácticas incluidas en el cuestionario; cada una de estas categorías es una variable dicotómica si/no.

En la Tabla 3 se adjunta un análisis descriptivo de las prácticas de RSC aplicadas por las empresas. Merece una mención especial el compromiso con el entorno y la preocupación por los stakeholders internos de la empresa (los trabajadores) que muestran las empresas encuestadas, pues más del 50\% aplica procedimientos de minimización de residuos en el origen y códigos de conducta. Sin embargo, los resultados muestran que en general los porcentajes de aplicación de las prácticas de RSC relacionadas con la gestión medioambiental ( $100 \%$ trabajos de recuperación en el cierre de explotaciones; $53 \%$ procedimientos de reducción de residuos en su origen; $53 \%$ control del consumo de fuentes de energía) superan a los de las prácticas relacionadas con acciones sociales y con el fomento de las comunidades locales. Este resultado no es nada sorprendente teniendo en cuenta que el impacto más directo de las actividades mineras recae sobre el entorno en el que operan, puesto que la extracción del mineral supone una alteración del medioambiente del que se obtiene. Precisamente durante las últimas décadas ha aumentado la preocupación por los impactos ambientales generados por la industria minera (Blinker, 2009) y las regulaciones gubernamentales e internacionales de las prácticas extractivas se han endurecido (Bruntland, 1987).

Tabla 3

Prácticas de RSC. En porcentaje de empresas

\begin{tabular}{|l|c|}
\hline $\begin{array}{l}\text { Trabajos de recuperación en el cierre } \\
\text { de explotaciones }\end{array}$ & $100 \%$ \\
\hline Códigos de conducta (o de buenas prácticas) & $60 \%$ \\
\hline $\begin{array}{l}\text { Procedimientos de reducción de residuos en su } \\
\text { origen }\end{array}$ & $53 \%$ \\
\hline Control del consumo de fuentes de energía & $53 \%$ \\
\hline Fomento económico de las comunidades locales & $40 \%$ \\
\hline Transparencia informativa & $37 \%$ \\
\hline Planes de carrera profesional & $13 \%$ \\
\hline Colaboración con ONG's & $6 \%$ \\
\hline
\end{tabular}

En la Tabla 4 se adjunta una síntesis de la aplicación de procedimientos de RSC y de los sistemas de medida. Los resultados muestran que prácticamente la mitad de las empresas no dispone de procedimientos de RSC ni de sistemas de medida de los resultados relacionados.

Se efectuaron contrastes de hipótesis para determinar si el nivel de formalización de procedimientos de gestión ética y sostenible que aplican las empresas está relacionado con la importancia que otorgan a la RSC, y si el grado de formalización de procedimientos de RSC está relacionado con la adopción de sistemas de medida de los resultados. Existe una relación significativa (al 5\%) entre el nivel de formalización de procedimientos de RSC y la utilidad percibida por las empresas (coeficiente de correlación 
de Spearman $=0,452)$. Existe una relación significativa (al 5\%) entre el nivel de formalización de procedimientos de RSC y el grado de aplicación de sistemas de medida de los resultados relacionados (coeficiente de correlación de Spearman =0,969).

Tabla 4

Procedimientos de RSC y sistemas de medida de los resultados. En porcentaje de empresas

\begin{tabular}{|l|c|c|}
\cline { 2 - 3 } \multicolumn{1}{c|}{} & $\begin{array}{c}\text { Procedimientos } \\
\text { (\%) }\end{array}$ & $\begin{array}{c}\text { Sistemas } \\
\text { de medida (\%) }\end{array}$ \\
\hline I: No en absoluto & 46 & 50 \\
\hline $\begin{array}{c}\text { 2: Si, para muy pocos ca- } \\
\text { sos }\end{array}$ & 20 & 16 \\
\hline $\begin{array}{c}\text { 3: Para el 50\% de los ca- } \\
\text { sos }\end{array}$ & 10 & 20 \\
\hline $\begin{array}{c}\text { 4: Si, para la mayoría de } \\
\text { los casos }\end{array}$ & 17 & 7 \\
\hline 5: Si, para todos los casos & 7 & 7 \\
\hline
\end{tabular}

Mediante el test de Kruskal-Wallis se determina que existe una relación significativa (al 5\%) entre la aplicación de un sistema de gestión medioambiental y la utilidad percibida de la RSC. La realización de prácticas vinculadas a la gestión medioambiental tiene una relación directa con la utilidad de la gestión ética y sostenible percibida por las empresas.

\section{Principales limitaciones del estudio}

El estudio de campo se limitó al territorio de Cataluña y a las empresas registradas a la base de datos del Gremio de Áridos de Cataluña. Si bien estas representan un porcentaje importante (60\% de las explotaciones mineras registradas en la comunidad autónoma de Cataluña), son un subsector concreto de la minería y no incluyen por tanto a todas las explotaciones en activo.

En investigaciones futuras cabría resolver estas limitaciones ampliando el análisis a otros subsectores mineros y a un ámbito territorial de mayor alcance.

\section{Conclusiones}

Puesto que el $57 \%$ de las empresas de la muestra tiene implantado un sistema de gestión de la calidad, puede afirmarse que las empresas del subsector minero de áridos de Cataluña están familiarizadas con los sistemas de gestión normalizados y documenta- dos, lo que puede facilitar la introducción de nuevos sistemas.

Sin embargo, sólo el 23\% dispone de un sistema de gestión medioambiental y el $3 \%$ ha implantado un sistema de gestión de la seguridad y salud laboral, siendo aspectos quizá no tan demandados por el cliente -como en el caso de la calidad- pero sí por el conjunto de la sociedad. Existe una extensa literatura sobre las ventajas de la implantación de dichos sistemas especialmente por lo que se refiere al cumplimiento de obligaciones legales.

Finalmente, la RSC aparece como la más desconocida pero hay evidencias de un incipiente interés, puesto que ya hay una empresa que tiene implantado un sistema de gestión.

Parece ser que el tamaño no es un factor decisivo para la implantación de sistemas de gestión. Este mismo resultado ya fue encontrado antes por distintos autores en el estudio de sistemas de gestión en la industria.

Uno de los aspectos que debe mejorarse es la gestión de las acciones de responsabilidad social. Únicamente el $24 \%$ de las empresas dispone de procedimientos de aplicación para la mayoría de las acciones de RSC y solamente el I $4 \%$ tiene establecidos sistemas de medida de los resultados derivados de estas acciones (considerando las puntuaciones 4 y 5 de la escala de Likert).

Existe una relación significativa entre el nivel de formalización de procedimientos de RSC y la utilidad percibida por las empresas. Existe una relación significativa entre el nivel de formalización de procedimientos de RSC y el grado de aplicación de sistemas de medida de los resultados relacionados.

Hay una relación significativa entre la aplicación de un sistema de gestión medioambiental y la utilidad percibida de la RSC. La realización de prácticas vinculadas a la gestión medioambiental tiene una relación directa con la utilidad de la gestión ética y sostenible percibida por las empresas. Este resultado se puede atribuir al elevado impacto que ocasionan las actividades mineras en el medioambiente. La presión legal en temas medioambientales explica que exista una concienciación por este tipo de problemas. La responsabilidad social también incluye aspectos de seguridad y salud en el trabajo que también están muy regulados. Sin embargo, los encuestados no parecen incluir este aspecto en su percepción de la responsabilidad social. 


\section{Referencias}

BANKS, G. (2006). Mining, social change and corporate social responsibility: Drawing lines in the Papua New Guinea mud. Globalisation, Governance and the Pacific Islands, pp. 259-274. Disponible en URL http://epress. anu.edu.au/ssgm/global_gov/mobile_devices/ch / 3.html [21/09/20l0].

BLINKER, L.R. (2009). Mining and the Natural Environment. UNCTAD, series of papers on Mining, Environment and Development, No.6, advanced copy. Disponible en URL http://www.unctad.org [21/09/2010].

BRUNTLAND, G. Ed. (1987). Our common future: The World Comission on Environment and Development. Oxford.

CARROLL, A.B. (1999). «Corporate Social Responsibility: Evolution of a definition construct». Business \& Society, 38 (3), pp. 268-295.

CASTILLA, M.L. (2003). Responsabilidad social corporativa: Tendencias empresariales en España. Madrid PricewaterhouseCoopers.

COWELL, S.J., WEHRMEYER, W., ARGUSTB, P.W. y ROBERTSON, J.G.S. (1999). «Sustainability and the primary extraction industries: theories and practice». Resource policy, 25 (4), pp. 277-286.

DEEGAN, C. y RANKIN, M. (1996). «Do Australian companies report environmental news objectively? An analysis of environmental disclosures by firms prosecuted successfully by the Environmental Protection Authority»>. Accounting, Auditing \& Accountability Journal, 9 (2), pp. 50-67.

ERKOREKA, J.I. (2006). Informe de la Subcomisión para potenciar y promover la RS de las empresas. Comisiones mixtas, subcomisiones y ponencias I54/000003. Boletín Oficial Cortes Generales. Congreso Diputados 423, pp. 3- 120.

FOMBRUN, C.y SHANLEY, M. ( 1990). «What's in a name? Reputation Building and Corporate Strategy». Academy of Management Journal, 33 (2), pp. 233-258.

GALÁN, J.I. (2008). «Responsabilidad social corporativa, cambio institucional y gobierno: introducción y panorámica». Revista Europea de Dirección y Economía de la Empresa, 17 (3), pp. 7.

GRI. (2005). Suplemento GRI del Sector Minería y Metales. Versión piloto 1.0. Disponible en URL http://www. globalreporting.org [21/09/20 I0].

GUERRA, M. (2004). «Community relations in mineral development projects». The CEPMLP Internet Journal, | I, pp. I-3।. Disponible en URL http://www.dundee. ac.uk/cepmlp/journal/html/voll I/voll I-6.html [2/ $/ 09 / 20$ l0].
HAMANN, R. (2004). «Corporate social responsibility, partnerships and institutional change». Natural Resources Forum, 28 (4), pp. 278-290.

HAMANN, R. y KAPELUS, P. (2004). «Corporate social responsibility in mining in Southern Africa: Fair accountability or just greenwash?». Development, 47 (3), pp. 8592.

HARRIS, N. (2007). «Corporate engagement in processes for planetary sustainability: understanding corporate capacity in the non-renewable resources extractive sector, Australia». Business Strategy and the Environment, 16, pp. 538-553.

ICMM. (2005). Reporting against the ICMM Sustainable Development Principles. International Council on Mining and Metals, London ICMM.

JENKINS, H. (2004). «Corporate social responsibility and the mining industry: conflicts and constructs». Corporate Social Responsibility and Environmental Management, II (I), pp. 23-34.

JENKINS, H. y YAKOVLEVA, N. (2006). «Corporate social responsibility in the mining industry: exploring trends in social and environmental disclosure». Journal of Cleaner Production, 14 (3-4), pp. 7|-284.

KAPELUS, P. (2002). «Mining, corporate social responsibility and the "Community": The Case of Rio Tinto, Richards Bay Minerals and the Mbonambi». Journal of Business Ethics, 39 (3), pp. 275-296.

KPMG. (2008). KPMG International survey of Corporate Responsibility Reporting 2008. Disponible en URL http://www.kpmg.com [21/09/20 l0].

LLORENS, F.J.; MOLINA, L.M. y FUENTES, M.M. (2002). «Calidad Total: Una investigación comparativa atendiendo a la dimensión empresarial». Investigaciones Europeas de Dirección y Economía de la Empresa, 8 (2), pp. | |9- 132.

MALHOTRA, M.J. y GROVER, V. (1998). «An assessment of survey research in POM: from constructs to theory». Journal of Operations Management, 16 (4), pp. 407-425.

MOGROVEJO, M.; PIMENTEL, R. y ZÚÑIGA, A. (2007). «Modelos de inversión social para empresas mineras. Experiencias y propuestas». Cuadernos de Difusión, 12 (23), pp. I I 5- 143.

NAVARRO, V. y DINIS, C. (2003). «Environmental, Health and Safety Management Systems for Underground Mining» en I st Int. Conf. on Sustainable Development and Management of the Subsurface, The Netherlands.

ORTEGA, E. (1990). Manual de Investigación Comercial. Madrid Ed. Pirámide.

SPENCE, L.J. (1999). «Does size matter? The state of the art in small business ethics》. Business Ethics: A European Review, 8 (3), pp. I63- 174. 
TÖREY, S. (2004). «La empresa minera: desde el blanco de las críticas hacia la acción pionera». Ambiente y Desarrollo, 20 (2), pp. 78-8I.

WALKER, J.y HOWARD, S. (2002).Voluntary codes of conduct in the mining industry. Mining, minerals and sustainable development project (MMSD), IIED, London Earthscan Publications Ltd.
WARHURST,A. (200I). «Corporate Citizenship and Corporate Social Investment: Drivers of Tri-Sector Partnerships». The Journal of Corporate Citizenship, I, pp. 57-70.

YAKOVLEVA, N. (2005). Corporate social responsibility in the mining industries. Hampshire Ashgate. 